\title{
Effects of cyclohexenonic long-chain fatty alcohol in type 2 diabetic rat nephropathy
}

\author{
Shinichi Okada ${ }^{1}$, Motoaki Saito ${ }^{2}$, Yukako Kinoshita ${ }^{2}$, Itaru $\mathrm{Satoh}^{2}$, Yasuo Kawaba ${ }^{1}$, Atsushi Hayashi ${ }^{1}$, \\ Takashi Oite ${ }^{3}$, Keisuke SATOH ${ }^{2}$, and Susumu KanZAKI ${ }^{1}$ \\ ${ }^{1}$ Division of Pediatrics and Perinatology, Tottori University Faculty of Medicine, 36-1 Nishi-cho, Yonago, 683-8504; ${ }^{2}$ Division of \\ Molecular Pharmacology, Tottori University Faculty of Medicine, 86 Nishi-cho, Yonago, 683-8503; and ${ }^{3}$ Department of Cellular Physi- \\ ology, Institute of Nephrology, Niigata University Graduate School of Medical and Dental Sciences, 1-757 Asahimachi-dori, Chuo-ku, \\ Niigata, 951-8150, Japan
}

(Received 13 April 2010; and accepted 14 May 2010)

\begin{abstract}
We attempted to clarify the effects of cyclohexenonic long-chain fatty alcohol (CHLFA) on the alterations of type 2 diabetes-induced nephropathy. Forty-week-old male Goto-Kakizaki (GK) and Wistar rats were divided into four groups of 6 to 8 animals. Group A consisted of eight Wistar rats and served as an age-matched control group. Group B (7 GK rats) received no treatment and served as a diabetic group. Group C (6 GK rats) was treated daily with low-dose CHLFA (2 mg/ $\mathrm{kg} /$ body weight, subcutaneously) for 30 weeks, and Group D (6 GK rats) with high-dose CHLFA $(8 \mathrm{mg} / \mathrm{kg} /$ body weight) for 30 weeks. At the end of the treatment period, urinary protein excretion, blood chemistry, renal histological, and immunohistological analyses were conducted. Although CHLFA administration did not influence serum glucose or insulin levels, it reversed diabetesinduced increases in urinary protein excretion and serum creatinine. Light microscopically, CHLFA treatment ameliorated the otherwise elevated glomerular sclerotic scores in the diabetic group. Immunohistochemically, increased expression of desmin and decreased expression of rat endothelial cell antigen-1 in the group with untreated diabetes both showed a reversal to control levels in the high-dose CHLFA treatment group. In conclusion, CHLFA may ameliorate type 2 diabetesinduced nephropathy.
\end{abstract}

Recently, diabetic nephropathy with type 2 diabetes has become the most common cause of end-stage renal disease. To inhibit the progression of diabetic vascular diseases, therapeutic agents such as reninangiotensin II system (RAS) inhibitors, radical scavengers (6) and PKC- $\beta$ inhibitors (12) have been investigated. However, in order to prevent diabetesinduced complications, the development of other

Address correspondence to: Motoaki Saito, MD, $\mathrm{PhD}$ Department of Pathophysiological and Therapeutic Science, Division of Molecular Pharmacology, Tottori University Faculty of Medicine, 86 Nishi-cho, Yonago 683-8503, Japan

Tel: +81-859-38-6162, Fax: +81-859-38-6160

E-mail: saitomo@med.tottori-u.ac.jp therapeutic agents will still be necessary.

The Goto-Kakizaki (GK) rat represents a spontaneous non-insulin-dependent diabetes model. GK rats are produced from normal Wistar rats by repeated selective breeding, and they are widely accepted as a genetically determined rodent model of human type 2 diabetes. This genetic rat model is particularly relevant for human type 2 diabetes research, because defects in glucose-stimulated insulin secretion, peripheral insulin resistance, and hyperinsulinemia are seen as early as four weeks after birth, and lateronset abnormalities include hypoinsulinemia and modest hyperglycemia (14).

The tropical plant, Hygrophilia erecta Hochr., has been shown to contain certain cyclohexenonic long-chain fatty alcohols (CHLFAs) that exhibit a 
neurotrophic activity on cultured neurons from the cerebral cortex $(2,3)$. CHLFA has been found to directly increase neurite extension as well as to enhance the biochemical differentiation of these neurons. We previously reported that CHLFA prevented the progression of diabetes-induced alterations in the trachea, aorta, and urinary bladder $(22$, 24, 25). Moreover, our previous reports demonstrated that CHLFA ameliorates the progression of diabetes-induced nephropathy in streptozotocin (STZ)induced diabetic rats $(17,21)$. Whereas CHLFA did not ameliorate the general features of diabetes, elevated serum glucose or decreased insulin levels in the STZ-induced diabetic rats, it did significantly prevent the progression of glomerulosclerosis. Based on the findings of these earlier studies, we propose that CHLFA may prevent not only type 1 diabetesinduced nephropathy, but also type 2 diabetesinduced nephropathy; furthermore, these preventive effects appear to take place in a manner independent of serum insulin and glucose levels.

Therefore, the aims of the present study were to investigate a feature of type 2 diabetes-induced nephropathy, and to clarify the possible ameliorative effects of CHLFA on type 2 diabetes-induced nephropathy.

\section{MATERIALS AND METHODS}

Animal models. All animal experiments were performed in accordance with the guidelines established by the Tottori University Committee for Animal Experimentation. Six-week-old male GK and Wistar rats were purchased from SLC (Shizuoka, Japan). All rats were kept under identical conditions, and all had access to food and drinking water ad libitum. At the age of 40 weeks, the rats were divided randomly into four groups $(n=7-8$ each): age-matched Wistar rats administered vehicle (A), diabetic GK rats administered vehicle (B), diabetic GK rats treated with cyclohexenonic long-chain fatty alcohol (CHLFA; 2,4,4-trimethyl-3-[15-hydroxypentadecyl]2-cyclohexen-1-one [Meiji Milk Products Co., Ltd., Tokyo, Japan]) at a daily dose of $2 \mathrm{mg} / \mathrm{kg}$ (C), and diabetic GK rats treated with CHLFA at a daily dose of $8 \mathrm{mg} / \mathrm{kg}$ (D) intramuscularly. CHLFA was dissolved in ethanol, and a mixture of physiological saline/Tween 80 was added at a ratio of ethanol: saline: Tween $80=5: 92.15: 2.85$ (total volume: $1 \mathrm{~mL} / \mathrm{kg}$ ). Drug or vehicle administration was initiated when each rat had reached the age of 40 weeks. Upon reaching 70 weeks of age, the rats were sacrificed with an overdose of pentobarbital
(60 mg intraperitoneally) after twenty-four hours of fasting. Immediately thereafter, blood samples were collected from the vena cava, and the renal tissues were removed. The blood samples and kidneys were frozen at $-80^{\circ} \mathrm{C}$ until use.

Measurement of urinary excretion and protein excretion. Over one 24-hour period between days 489 and 490 (week 70), the rats were placed in a metabolic cage containing a urine-collection funnel over an electronic balance (HL200; A.N.D., Tokyo, Japan) to measure urine excretion. Sesame oil (Nisshin OilliO Group, Ltd., Tokyo, Japan) was used to prevent evaporation of the urine during collection. The balances were connected to a personal computer (Macintosh Power Book G4; Apple Computer, Cupertino, CA) via a multiport controller (PowerLab/ $8 \mathrm{sp}$; AD Instruments, Castle Hill, Australia) to monitor the cumulative weight of the collected urine. All rats received food and water ad libitum in the cage. Each monitoring period started at $18: 00$, and urine was collected for exactly $24 \mathrm{~h}$. Then, the total urine excretion of the rats was determined. After the urine of each animal had been collected, the urinary levels of protein were determined using an enzymelinked immunosorbent assay (ELISA) kit (Protein Assay Rapid Kit Wako; Wako Pure Chemical Industries, Osaka, Japan) according to the manufacturer's instructions.

Serum glucose, insulin, creatinine, and blood urea nitrogen measurement. The serum glucose, insulin, creatinine, and blood urea nitrogen concentrations of experimental rats were measured by the hexokinase method (Glucose C II; Wako Pure Chemical Industries), the ELISA method (Rat Insulin ELISA; Mercodia AB, Uppsala, Sweden), the Jaffe method (Creatinine-test Wako; Wako Pure Chemical Industries), and the urease-indophenol method (Urea N B; Wako Pure Chemical Industries), respectively, all of which were carried out according to the kit manufacturers' instructions.

Histological examination of the rat kidney. The right kidney was immediately fixed with $10 \%$ formalin. After fixation, the tissues were embedded in paraffin, and $5-\mu \mathrm{m}$-thick tissue sections were cut from the paraffin blocks. All of the kidney specimens were stained using hematoxylin and eosin (H \& E) and periodic acid-Schiff (PAS). Each section was viewed under a light microscope at a magnification of $\times 100-400$. Histological evaluation was performed without knowledge of the identity of the various 
groups. In the PAS-stained sections, approximately 100 glomeruli in a specimen $(n=8,7,7$, or 7 per group, respectively) were evaluated by one pathologist and one nephrologist, and mesangial matrix expansion and glomerular sclerosis were graded semiquantitatively on a scale of 0 to $4+$ according to the method described by Raij et al. (19). The areas used for evaluation were randomly selected, and the values were expressed as per high-powered field (HPF). The numbers of glomeruli with nodule-like lesions were counted, and these values were normalized with the number of glomeruli counted.

Immunohistochemical analysis of the rat kidney. The serial sections of the kidney were also subjected to immunohistochemical staining for $\alpha$-smooth muscle cell actin ( $\alpha$-SMA), desmin, ED-1, and collagen type IV. Briefly, after the sections were deparaffinized, they were then reacted overnight with anti$\alpha$-SMA, anti-desmin (Abcam Ltd., Cambridge, UK), anti-ED-1 (AbD Serotec Ltd., Kidlington, UK) or anti-collagen type IV polyclonal primary antibody (Progen Biotechnik GmbH, Heidelberg, Germany) at a $1: 100,1: 200,1: 100$ or $1: 50$ dilution, respectively. The specifically bound first antibodies were visualized by a secondary antibody conjugated with peroxidase using Histofine simple stain (rat MAX-PO) (Nichirei Co., Tokyo, Japan) with a Histofine simple stain substrate (Nichirei Co.). Mayer's hematoxylin was used for counterstaining. Negative controls were established for each group according to the procedure of immunohistochemical staining mentioned above, but without a secondary antibody conjugated with peroxidase. Immunohistochemical evaluation was performed without knowledge of the identity of the various groups. In each of the stained sections, approximately 100 randomly selected glomeruli or a field of 20 tubulointerstitial areas were analyzed in each specimen (group A, B, C and D: $n=8,7,7$ and 7 , respectively) by one pathologist and one nephrologist, and immunostaining of the glomerular area and tubulointerstitial grid field were graded semiquantitatively on a scale of 0 to $4+$ according to the method of Janssen et al. (7).

Immunofluorescent microscopy of the rat kidney using frozen sections. To assess the glomerular nodulelike lesions, immunofluorescent double-label staining for platelet-endothelial cell adhesion molecule-1 (PECAM-1; CD-31) and Thy1 antigen (OX-7) was evaluated in group B. Frozen tissue sections were incubated with mouse monoclonal antibody against rat PECAM-1(Serotec Ltd., Kidlington, UK) as a primary antibody, followed by incubation with FITC-labeled rabbit anti-mouse immunoglobulins (Dako Denmark A/S, Glostrup, Denmark) as a secondary antibody. Then, the sections were incubated with biotinylated mouse antibody against rat OX-7 (Abcam) for identifying glomerular mesangial cells, followed by incubation with tetramethyl rhodamine $\mathrm{B}$ isothiocyanate-conjugated streptavidin (KPL Inc., Gaithersburg, MD). Stained sections were examined with confocal laser scanning immunofluorescent microscopy (MRC-1024; Bio-Rad Laboratories, Hemel Hempstead, UK).

Serial sections of tissue were also subjected to immunofluorescent double-label staining for rat endothelial cell antigen-1 (RECA-1) and Thyl antigen (OX-7) for further evaluation. Monoclonal mouse anti-RECA-1 antibody was obtained from AbD Serotec. The secondary antibodies were FITC-conjugated rabbit anti-mouse immunoglobulin (Dako Denmark A/S) for the anti-RECA-1 antibody, and TRITC-conjugated anti-mouse immunoglobulin (Dako Denmark A/S) for the anti-OX-7 antibody. In the immunofluorescent-stained sections, approximately 3 to 13 glomeruli in a specimen $(n=4,4,4$, or 3 per group, respectively) were evaluated by one pathologist and one nephrologist, and the expansion scores based on immunofluorescent staining were graded semiquantitatively on a scale of 0 to $4+$.

Data analysis. All data were evaluated by parametric ANOVA and expressed as the means \pm S.E.M. Statistical analyses were conducted using the TukeyKramer Test for multiple comparisons and Kyplot software. Student's $t$-test was used for comparison of urinary albumin excretion and for biochemical serum analyses. For all statistical tests, a $P$-value of less than 0.05 indicated statistical significance.

\section{RESULTS}

\section{General features of experimental rats}

General features of each group are shown in Table 1. By the age of 40 weeks, GK diabetic rats showed a significantly smaller amount of weight gain than their non-diabetic counterparts, and these rats also showed no weight gain during the experimental period. The kidney weight and the kidney weight/body weight ratio of GK diabetic rats significantly increased. Treatment with CHLFA neither increased the body weight gain nor decreased the kidney weight/body weight ratio. Moreover, GK diabetic rats had significantly higher serum glucose levels and lower serum insulin levels than control rats (Ta- 
ble 2). Treatment with CHLFA had no effect on either serum glucose or insulin levels. The GK diabetic rats also exhibited increased levels of serum creatinine and urinary protein excretion, as compared to those of control rats. CHLFA had no effect on blood urea nitrogen levels, but high-dose $(8 \mathrm{mg} /$ $\mathrm{kg}$ ) CHLFA improved serum creatinine and urinary protein excretion levels compared to those of untreated GK rats (Table 2).

\section{Histological examination of the rat kidney}

Fig. 1 shows PAS-stained specimens of all groups, and Fig. 2 shows the mesangial matrix expansion scores and glomerular sclerosis scores. In the untreated GK rat group (group B), mesangial matrix expansion and segmental glomerular sclerosis were evident. The mesangial matrix expansion scores in Figs. 1A, B, C, and D were $1+, 4+, 2+$, and $1+$, respectively. On the other hand, the glomerular sclerosis scores in Figs. 1A, B, C, and D were 0, 3+, 1+, and $1+$, respectively. Treatment with CHLFA significantly ameliorated diabetes-induced alteration of the kidney. The mesangial matrix expansion scores and glomerular sclerosis scores of untreated GK rats (group B) were significantly higher than those of control rats (group A) (Fig. 2). Although the findings did not reach the level of statistical signifi- cance, CHLFA appeared to inhibit the progression of glomerular sclerosis in a dose-dependent manner and tended to reverse mesangial matrix expansion.

Glomeruli with segmental nodule-like lesions (Figs. 1B, 1C, and 1D) similar to nodular Kimmelstiel-Wilson lesions were observed in all groups. The scores in the untreated GK rats (group B) were significantly higher than those of the control group (Fig. 3). Although the association was not statistically significant, CHLFA tended to be associated with scores approaching normal levels.

\section{Immunohistochemical analysis of the rat kidney}

Immunostaining for $\alpha$-SMA, a marker of activated mesangial cells (8), in the glomeruli did not differ among groups (Fig. 4). The glomerular expression of desmin, a marker of injured podocytes $(4,32)$, was significantly higher in untreated GK rats (group B) than in control rats (group A), and CHLFA inhibited the expression of desmin in a dose-dependent manner (Figs. 4 and 6). Fig. 6B shows an accumulation of desmin in the podocyte region, and this accumulation was significantly lower in group D. Although the findings did not reach the level of significance, the expression of ED-1, a marker of monocyte/macrophage infiltration into glomeruli, was upregulated in untreated GK rats (group B),

Table 1 The general features in the experimental rats

\begin{tabular}{cccccc}
\hline & \multicolumn{2}{c}{ Body weight $(\mathrm{g})$} & Kidney weight $(\mathrm{g})$ & $\begin{array}{c}\text { Kidney weight }(\mathrm{mg}) / \\
\text { body weight }(\mathrm{g})\end{array}$ \\
\cline { 1 - 3 } & Age 40 weeks & Age 70 weeks & & $1.40 \pm 0.04$ & $2.58 \pm 0.06$ \\
$\mathrm{~A}$ & $535.6 \pm 19.8$ & $546.3 \pm 20.0$ & & $1.59 \pm 0.04^{*}$ & $4.01 \pm 0.08^{*}$ \\
$\mathrm{~B}$ & $419.3 \pm 4.0^{*}$ & $394.3 \pm 3.7^{*}$ & & $1.65 \pm 0.06^{*}$ & $4.18 \pm 0.14^{*}$ \\
$\mathrm{C}$ & $416.4 \pm 8.3^{*}$ & $400.0 \pm 8.7^{*}$ & & $1.53 \pm 0.05$ & $3.96 \pm 0.16^{*}$ \\
\hline
\end{tabular}

A: control rats, B: diabetic rats, C: diabetic rats treated with $2 \mathrm{mg} / \mathrm{kg}$ cyclohexenonic long-chain fatty alcohol (CFLFA), and D: diabetic rats treated with $8 \mathrm{mg} / \mathrm{kg}$ CHLFA.

Data are shown as mean \pm S.E.M. of six to eight separated determinations in each group.

* Significantly different from group A. $(P<0.05)$

Table 2 The experimental data in the rats

\begin{tabular}{ccccccc}
\hline & $\begin{array}{c}\text { Serum glucose } \\
(\mathrm{mg} / \mathrm{dL})\end{array}$ & $\begin{array}{c}\text { Serum insulin } \\
(\mu \mathrm{g} / \mathrm{L})\end{array}$ & $\begin{array}{c}\text { Urinary excretion } \\
(\mathrm{mL} / \mathrm{day})\end{array}$ & $\begin{array}{c}\text { Serum BUN } \\
(\mathrm{mg} / \mathrm{dL})\end{array}$ & $\begin{array}{c}\text { Serum creatinine } \\
(\mathrm{mg} / \mathrm{dL})\end{array}$ & $\begin{array}{c}\text { Protein excretion } \\
(\mathrm{mg} / 24 \mathrm{~h})\end{array}$ \\
\hline $\mathrm{A}$ & $132.2 \pm 5.9$ & $2.51 \pm 0.33$ & $12.7 \pm 1.3$ & $21.8 \pm 1.9$ & $0.78 \pm 0.09$ & $88.7 \pm 23.4$ \\
$\mathrm{~B}$ & $208.1 \pm 8.5^{*}$ & $0.53 \pm 0.10^{*}$ & $13.2 \pm 1.5$ & $22.7 \pm 1.3$ & $0.99 \pm 0.04^{*}$ & $221.1 \pm 38.4^{*}$ \\
$\mathrm{C}$ & $217.3 \pm 16.4^{*}$ & $0.54 \pm 0.10^{*}$ & $14.1 \pm 2.9$ & $24.0 \pm 1.0$ & $0.99 \pm 0.08^{*}$ & $209.4 \pm 24.5^{*}$ \\
$\mathrm{D}$ & $209.1 \pm 6.1^{*}$ & $0.94 \pm 0.15^{*}$ & $15.3 \pm 1.3$ & $20.8 \pm 1.1$ & $0.92 \pm 0.05$ & $171.0 \pm 30.4$ \\
\hline
\end{tabular}

A: control rats, B: diabetic rats, C: diabetic rats treated with $2 \mathrm{mg} / \mathrm{kg}$ cyclohexenonic long-chain fatty alcohol (CHLFA), and D: diabetic rats treated with $8 \mathrm{mg} / \mathrm{kg}$ CHLFA.

Data are shown as mean \pm S.E.M. of six to eight separated determinations in each group.

*Significantly different from group A. $(P<0.05)$ 

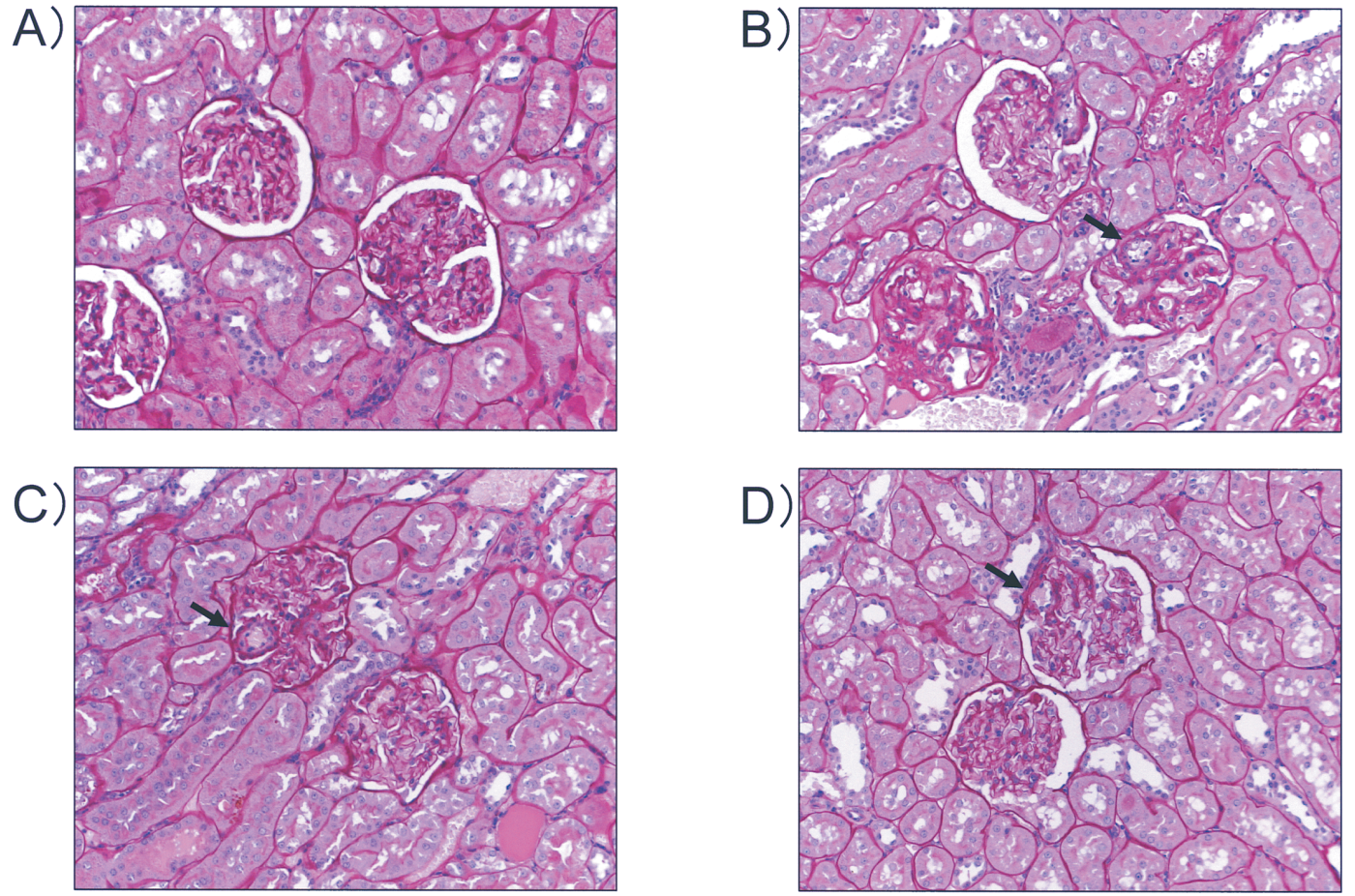

Fig. 1 Histological examination of the kidney. A: Wistar rats, B: GK rats treated with vehicle, C: GK rats treated with 2 mg/ $\mathrm{kg}$ of cyclohexenonic long-chain fatty alcohol (CHLFA), and D: GK rats treated with $8 \mathrm{mg} / \mathrm{kg}$ of CHLFA. Severe mesangial matrix expansion and segmental glomerular sclerosis are observed in the untreated GK rat group (B). Glomeruli with segmental nodule-like lesions are observed (arrows). (×400 magnification)
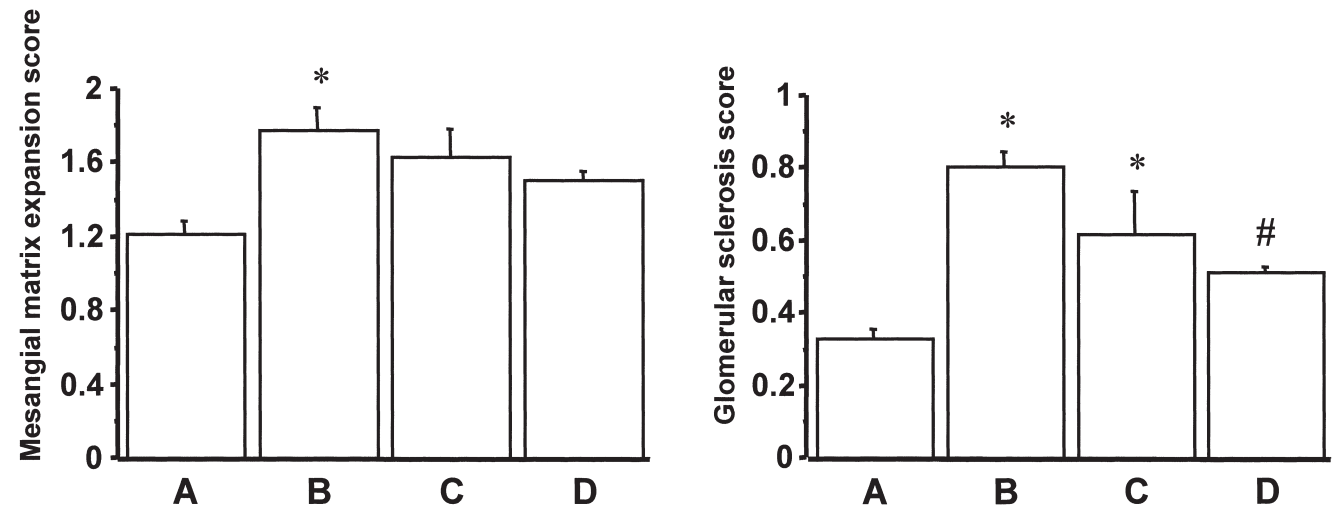

Fig. 2 Histological evaluation of the kidney for mesangial matrix expansion score and glomerular sclerosis score. A: Wistar rats, B: GK rats treated with vehicle, C: GK rats treated with $2 \mathrm{mg} / \mathrm{kg}$ of cyclohexenonic long-chain fatty alcohol (CHLFA), and D: GK rats treated with $8 \mathrm{mg} / \mathrm{kg}$ of CHLFA. Data are shown as the means \pm S.E.M. of five separate determinations in each group. *: Significantly different compared to group A $(P<0.05)$. ": Significantly different compared to group $\mathrm{B}(P<0.05)$.

and tended to be lower in groups $\mathrm{C}$ and $\mathrm{D}$ in a dose-dependent manner (Fig. 4). On the other hand, glomerular collagen type IV expression did not differ among the groups (Fig. 4).

In the renal tubules and the peritubular interstitium, the levels of expression of $\alpha$-SMA, a marker of myofibroblasts in the interstitium, and ED-1, a marker of monocyte/macrophage infiltration into the interstitium in the untreated GK rats (group B) tended to increase as compared to those of control rats (group A) (Fig. 5). In contrast, the level of collagen type IV, a marker of fibrosis of the interstitium, in the untreated GK rats (group B) significantly increased as compared to those of control rats (group 
A) (Fig. 5). Treatment with high-dose CHLFA decreased the expression of these markers, albeit not significantly. Low levels of desmin expression were seen in all groups (data not shown).

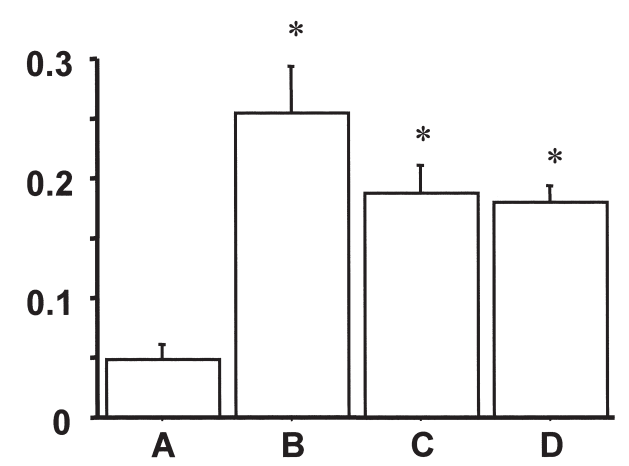

Fig. 3 Histological evaluation of nodule-like lesions. A: Wistar rats, B: GK rats treated with vehicle, C: GK rats treated with $2 \mathrm{mg} / \mathrm{kg}$ of cyclohexenonic long-chain fatty alcohol (CHLFA), and D: GK rats treated with $8 \mathrm{mg} / \mathrm{kg}$ of CHLFA. Data are shown as the means \pm S.E.M. of five separate determinations in each group. *: Significantly different compared to group $\mathrm{A}(P<0.05)$.
Immunofluorescent microscopic analysis of frozen sections of the rat kidney

Anti-CD-31 antibody and anti-OX-7 antibody respectively reacted with endothelial cells of the glomerular capillary and mesangial cells. Positive staining with both antibodies was observed in the glomeruli (Fig. 7). CD-31 staining in the glomeruli surrounded nodule-like lesions, and negative internal staining of the nodule-like lesions was also observed (Fig. 7). On the other hand, OX-7-positive staining was observed in the nodule-like lesions, as shown in the merged images (Fig. 7).

Fig. 8 shows the results of immunostaining for RECA-1 or OX-7 in all groups, and Fig. 9 shows the expansion scores based on immunofluorescent stainings. Immunostaining for RECA-1 in untreated GK rats (group B) (Fig. 8d) revealed that the number of RECA-1-positive capillaries decreased as compared to that in control rats (group A) (Fig. 8a), and capillary density had recovered to normal in group D (Figs. 8j and 9), although neither result was statistically significant. On the other hand, immunostaining for OX-7 in untreated GK rats (group B)
$\alpha-S M A$

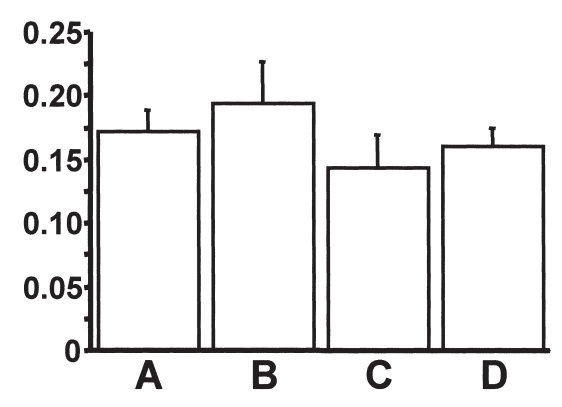

ED-1

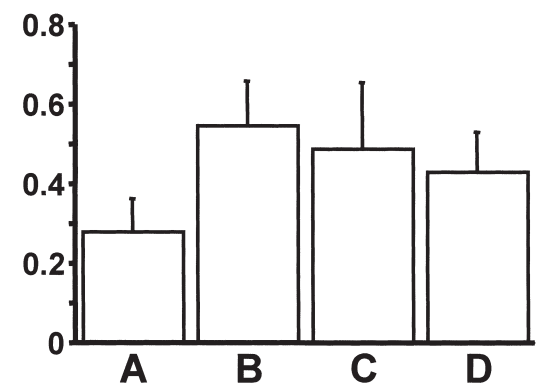

desmin

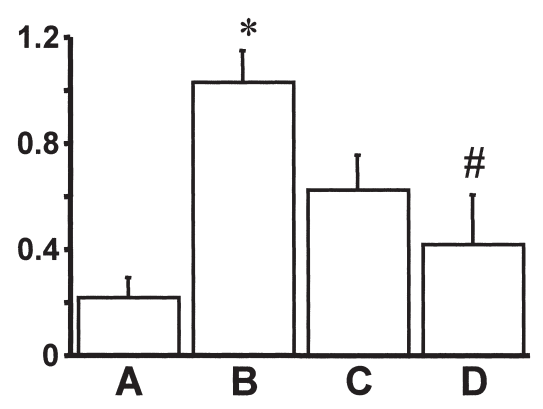

collagen type IV

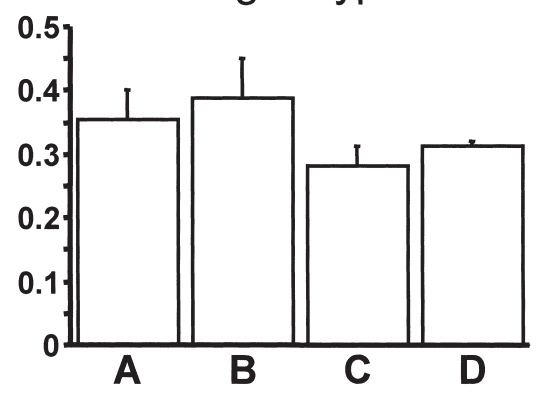

Fig. 4 Immunohistochemical scoring of the glomeruli for $a$-smooth muscle cell actin ( $a$-SMA), desmin, ED-1, and collagen type IV. A: Wistar rats, B: GK rats treated with vehicle, C: GK rats treated with $2 \mathrm{mg} / \mathrm{kg}$ of cyclohexenonic long-chain fatty alcohol (CHLFA), and D: GK rats treated with $8 \mathrm{mg} / \mathrm{kg}$ of CHLFA. Data are shown as the means \pm S.E.M. of three separate determinations in each group. *: Significantly different compared to group $A(P<0.05)$. ": Significantly different compared to group $\mathrm{B}(P<0.05)$. 
$\alpha-S M A$

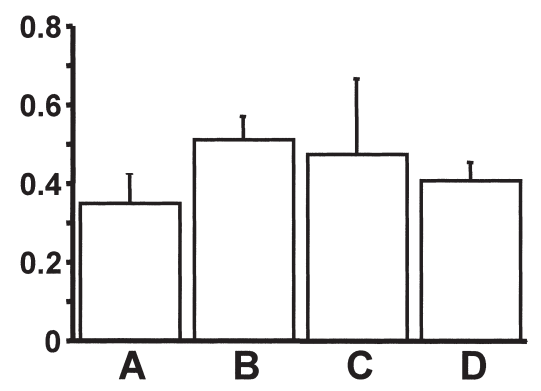

collagen type IV

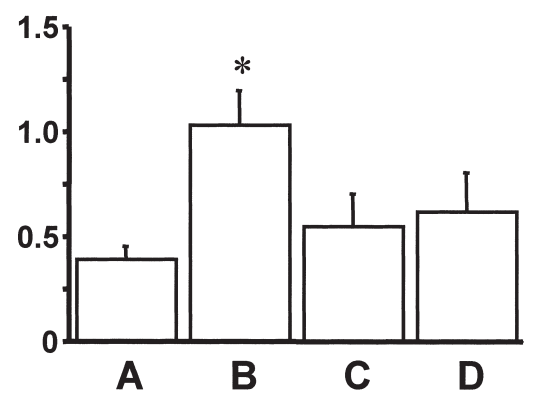

ED-1

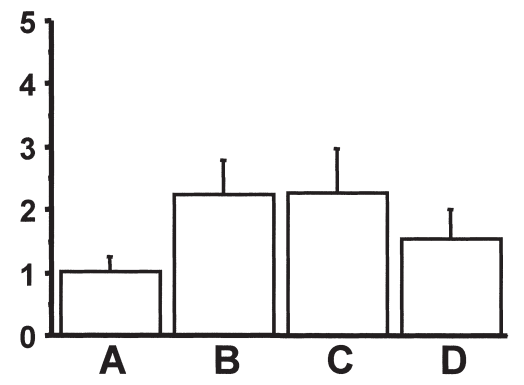

Fig. 5 Immunohistochemical evaluation of renal tubules and peritubular interstitium for $a$-smooth muscle cell actin ( $a$-SMA), ED-1, and collagen type IV. A: Wistar rats, B: GK rats treated with vehicle, C: GK rats treated with $2 \mathrm{mg} / \mathrm{kg}$ of cyclohexenonic long-chain fatty alcohol (CHLFA), and D: GK rats treated with $8 \mathrm{mg} / \mathrm{kg}$ of CHLFA. Data are shown as the means \pm S.E.M. of three separate determinations in each group. *: Significantly different compared to group $\mathrm{A}(P<0.05)$.

A)

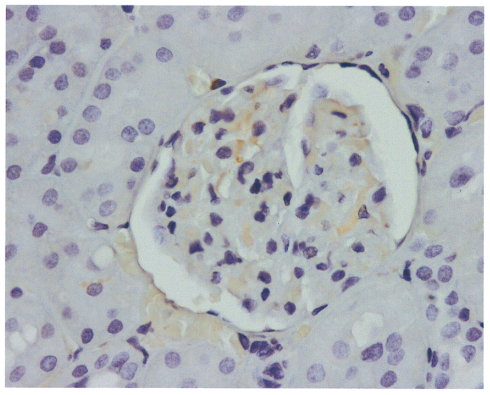

C)

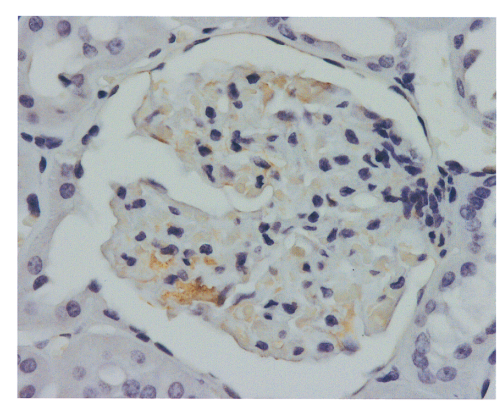

B)

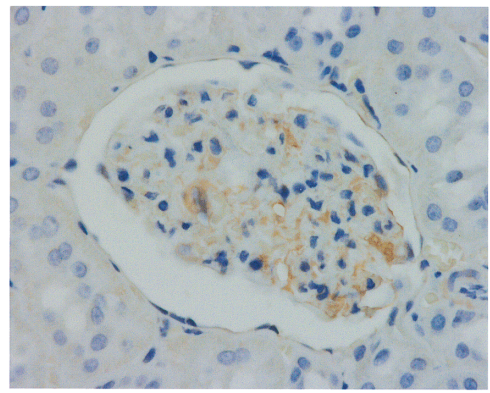

D)

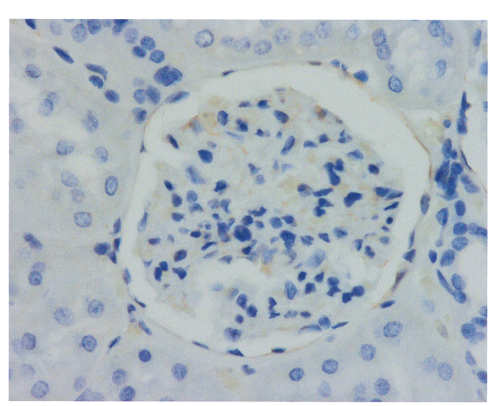

Fig. 6 Immunohistochemical examination of the glomeruli for desmin. A: Wistar rats, B: GK rats treated with vehicle, C: GK rats treated with $2 \mathrm{mg} / \mathrm{kg}$ of cyclohexenonic long-chain fatty alcohol (CHLFA), and D: GK rats treated with $8 \mathrm{mg} / \mathrm{kg}$ of CHLFA. Desmin expression mostly exhibited an endocapillary pattern and was up-regulated in the untreated GK rat group (B). ( $\times 400$ magnification) 
CD-31

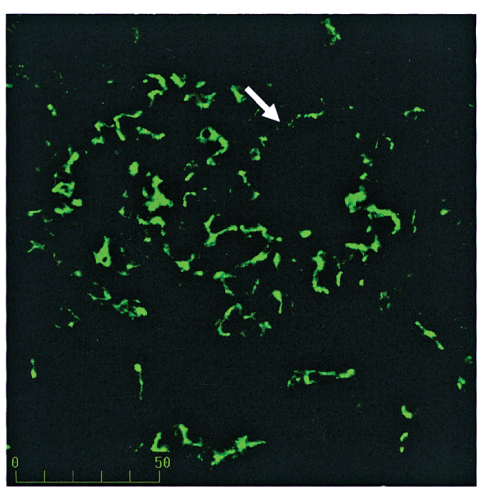

merged

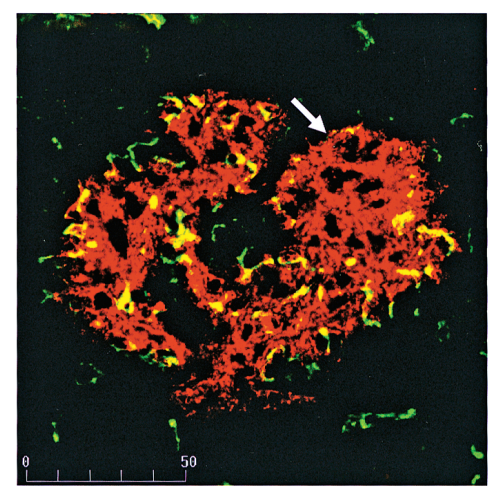

OX-7

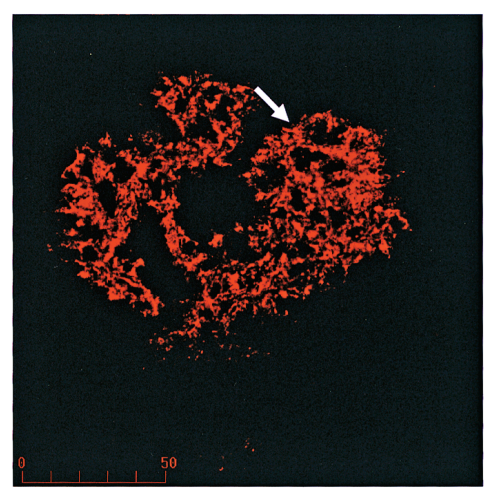

Fig. 7 Immunofluorescent microscopy of the untreated GK rat kidney for CD-31 and OX-7. Nodule-like lesions were observed (arrows).

(Fig. 8f) tended to be more intense than that of control rats (group A) (Fig. 8c), and normal immunostaining intensities were observed in group D (Figs. 81 and 9), although neither result was significant.

\section{DISCUSSION}

In this study, we investigated the effects of CHLFA on spontaneous type 2 diabetes-induced nephropathy using a rat model. Treatment with CHLFA did not alter diabetic status-e.g., body weight, urinary excretion, serum glucose, or insulin or blood urea nitrogen levels - but it ameliorated high urinary protein excretion and serum creatinine levels. Treatment with CHLFA also inhibited diabetes-induced increase in kidney weight. CHLFA reduced the glomerular matrix expansion, inhibited the progression of glomerular sclerosis, and improved the glomerular expression of desmin. Our data indicate that CHLFA could prevent type 2 diabetes-induced nephropathy.

The GK rat represents a spontaneous non-insulindependent diabetes model. GK rats are produced from normal Wistar rats by repetitive selective breeding, and are widely accepted to be a genetically determined rodent model for human type 2 diabetes. This genetic rat model exhibits a modest degree of renal dysfunctional or structural changes, in addition to the development of glomerular basement membrane (GBM) thickening in animals at 8 to 12 weeks (27). Nobrega and co-workers have also reported that GK rats exhibited a thickening of the GBM, mild mesangial matrix expansion, and glomerular hypertrophy (16). When age-induced progression of glomerular sclerosis was evaluated, glomerular sclerosis of 24-month-old GK rats was more severe than that of 8- or 12-month-old GK rats (23). In type 2 diabetes-induced nephropathy, including that in GK rats, exposure to a prolonged diabetic state occurs.

In the present study, we demonstrated that GK rats exhibited mesangial matrix expansion, segmental glomerular sclerosis, and nodule-like lesions; to our knowledge, few of the previous reports on diabetes-induced nephropathy in GK rats have shown the existence of nodule-like lesions. This discrepancy might be due to differences in the strains of GK rats used or in the durations of the diabetic state. In this study, the nodule-like lesions showed positive staining for OX-7, a mesangial marker, and an absence of staining for PECAM-1, an endothelial marker. These findings indicate that the nodule-like lesions observed here consisted of mesangial matrix. Kimmelstiel-Wilson nodules consist of mesangial matrix and endothelial cells (28), and show positive staining for OX-7 and PECAM-1. Therefore, Kimmelstiel-Wilson nodules differ from the nodule-like lesions observed in the present study, which lacked PECAM-1 expression. In diabetic nephropathy, mesangial extracellular matrix accumulates in the glomeruli, leading to glomerular sclerosis. The overall accumulation of mesangial extracellular matrix is regulated by the relative levels of synthesis and degradation of the matrix, and is determined by a balance between proteases and inhibitors (29). Because CHLFA reduced mesangial matrix expansion, CHLFA may exert its effects on proteases or inhibitors, or both. Further examination of this issue will be necessary in future studies.

Hyperglycemia plays an important role in the development of diabetic nephropathy. Although treatment with CHLFA was not found to alter serum 

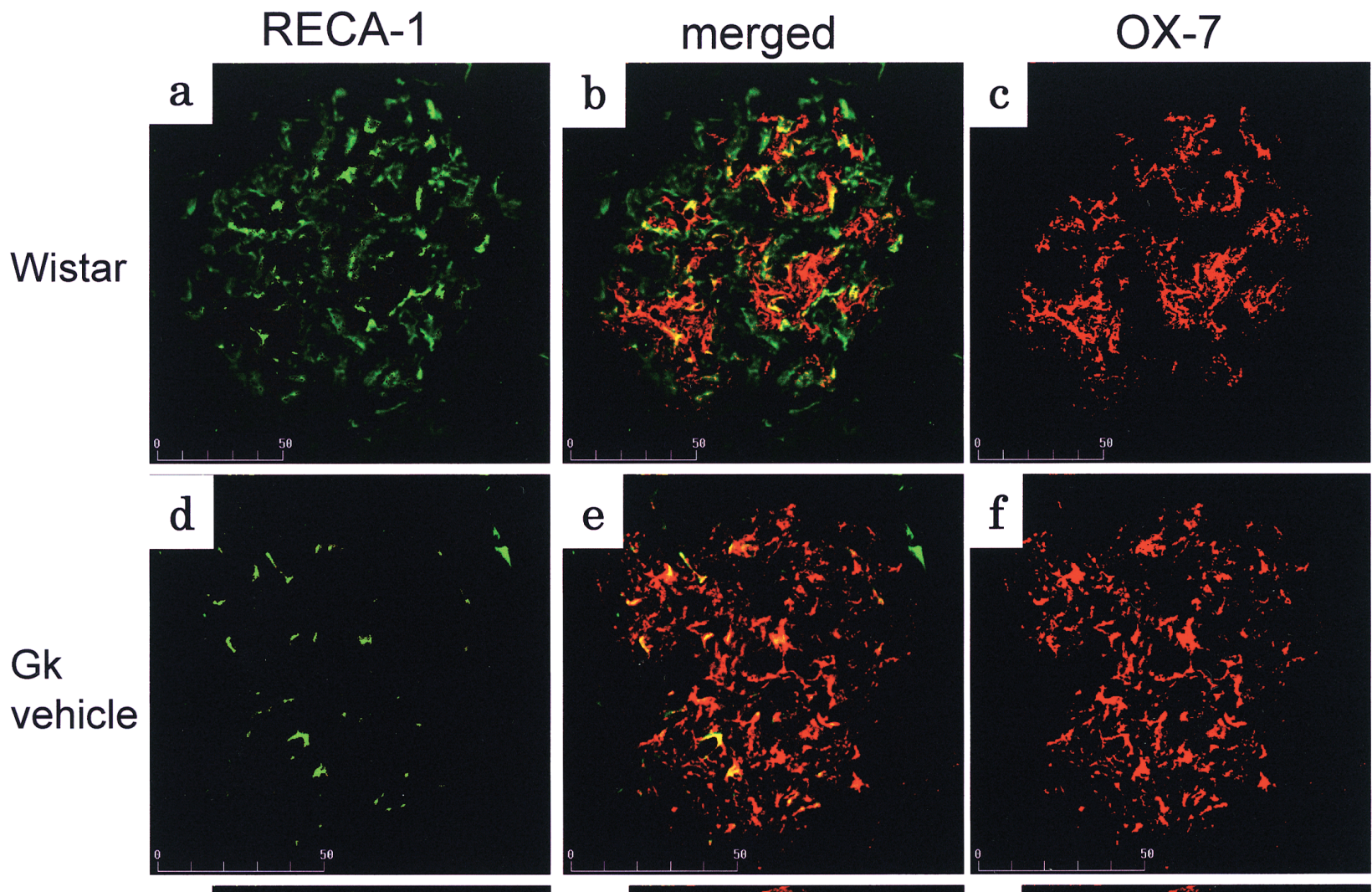

e
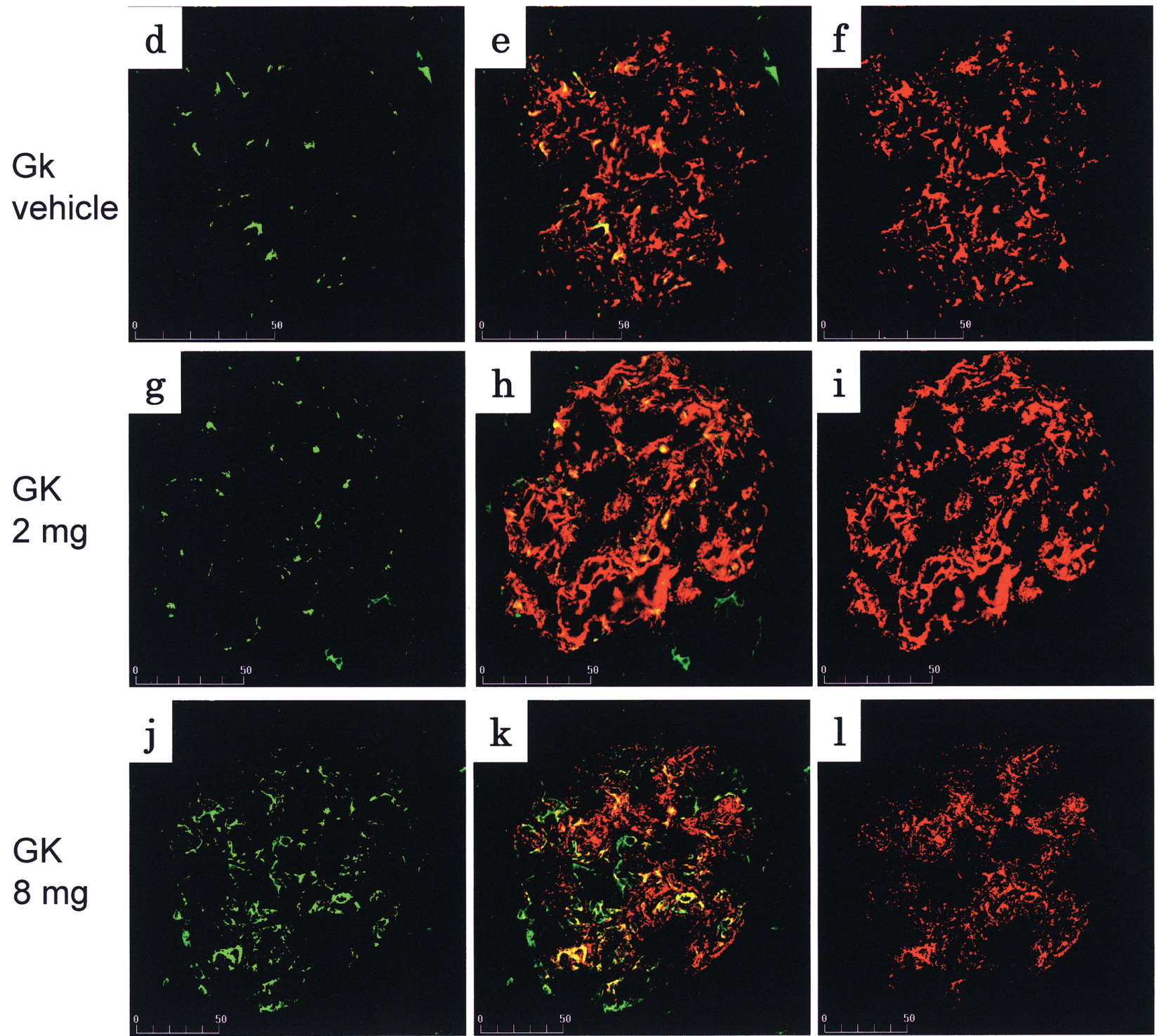

Fig. 8 Immunofluorescent microscopy of the rat kidney for rat endothelial cell antigen-1 (RECA-1) and OX-7. Wistar: Wistar rats (group A), GK vehicle: GK rats treated with vehicle (group B), GK $2 \mathrm{mg}$ : GK rats treated with $2 \mathrm{mg} / \mathrm{kg}$ of cyclohexenonic long-chain fatty alcohol (CHLFA) (group C), and GK $8 \mathrm{mg}$ : GK rats treated with $8 \mathrm{mg} / \mathrm{kg}$ of CHLFA (group D). The panels a, $\mathbf{d}, \mathbf{g}$, and $\mathbf{j}$ show immunostaining for RECA-1 antibodies. The panels $\mathbf{c}, \mathbf{f}$, $\mathbf{i}$, and $\mathbf{I}$ show immunostaining for OX-7 antibodies. The panels $\mathbf{b}, \mathbf{e}, \mathbf{h}$, and $\mathbf{k}$ show merged images. 


\section{RECA-1}

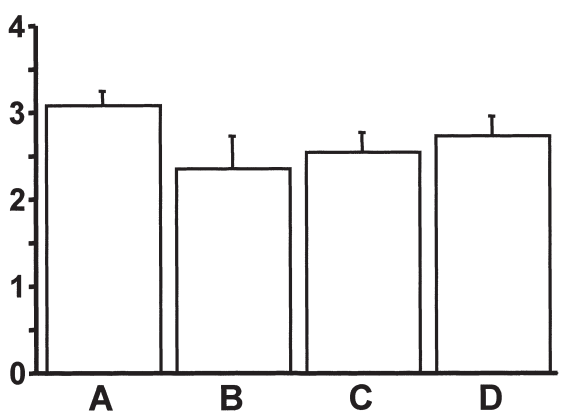

OX-7

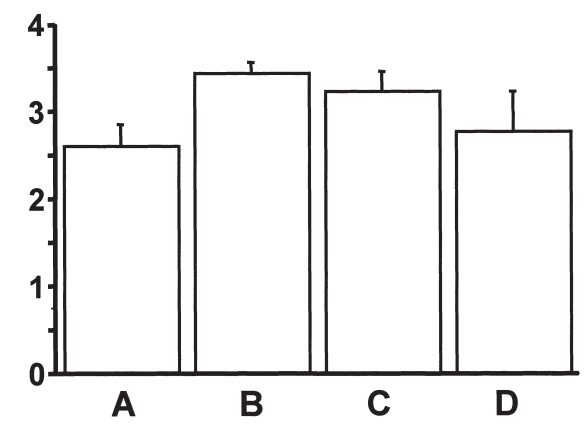

Fig. 9 Immunofluorescent analysis of the kidney for expressions of rat endothelial cell antigen-1 (RECA-1) and OX-7. A: Wistar rats, B: GK rats treated with vehicle, C: GK rats treated with $2 \mathrm{mg} / \mathrm{kg}$ of cyclohexenonic long-chain fatty alcohol (CHLFA), and D: GK rats treated with $8 \mathrm{mg} / \mathrm{kg}$ of CHLFA. Data are shown as the means \pm S.E.M. of five separate determinations in each group.

glucose levels in the present study, it did prevent the progression of diabetic nephropathy similar to that caused by type 1 diabetes $(17,21)$. It is known that an angiotensin II blockade prevents the progression of diabetic nephropathy without altering glucose levels; CHLFA may exert its effects in a similar manner, although the precise mechanism of such effects, including those at the molecular level, remains unclear at present.

Recently, it has been reported that inhibition of sympathetic nerve activity reduced albuminuria in patients with chronic kidney disease (13), and ameliorated glomerulosclerosis in subtotally nephrectomized rats (1). Another study reported that, by reducing the increased activities of renal sympathetic nerves, the renal efferent arteries could be vasodilated, leading to an improvement in glomerular hypertension (31). It has been reported that CHLFA affects extensions of neurites and differentiations of neurons $(2,3)$. Watanabe and Miyagawa reported that CFLFA has beneficial effects on peripheral neuropathy and cystopathy in STZ-induced diabetic rats (26). Such effects of CHLFA on neurites and neurons may be one of the mechanisms involved in the alterations of diabetic nephropathy.

The progression of diabetes-induced complications is correlated with endothelial dysfunction, which in turn plays an important role in the progression of diabetic nephropathy $(9,30)$. Nakagawa and co-workers reported that endothelial NO synthaseknockout mice under diabetic conditions exhibit severe diabetic nephropathy (15). As endothelial cells regulate mesangial cell proliferation (20), diabetesinduced endothelial dysfunction induces mesangial cell injury, in turn leading to mesangial matrix ex- pansion. In this study, high-dose CHLFA treatment was associated with a recovery of capillary density, as shown by immunofluorescence analysis. In our previous reports, we have shown that CHLFA inhibits the progression of diabetes-induced endothelial dysfunction of the aorta in rats with STZ-induced diabetes $(10,25)$. Here, CHLFA improved diabetesinduced endothelial dysfunction, resulting in the amelioration of diabetic nephropathy.

Podocyte injuries are observed in association with diabetic nephropathy (18), and it is known that injured podocytes express desmin $(4,32)$. In the present study, diabetic GK rats showed an increased expression of desmin in the glomeruli, and CHLFA reduced this increase. The mechanisms of this effect were not clear, but it is possible that CHLFA exerts direct effects on glomerular podocyte processes, much as it affects neurons (5). It has been reported that the characteristics of podocyte processes closely resemble those of neuronal dendrites. In both podocyte primary processes and neurites, microtubules and intermediate filaments are major cytoskeletal components, and the microtubules contribute to establishment of the mixed polarity in the podocyte primary processes and neurites. Furthermore, the elongation of podocyte processes and neurites is regulated by the same mechanisms as used for membrane transport (11). Therefore, CHLFA may ameliorate diabetes-induced podocyte injury via mechanisms similar to the effects it exerts on neurons, although further investigations will be needed to examine these issues in more detail.

In conclusion, CHLFA was shown to ameliorate type 2 diabetes-induced nephropathy, just as it ameliorates nephropathy caused by type 1 diabetes. 


\section{Acknowledgements}

This work was published in abstract form at the World Congress of Nephrology (Milan 2009). This study was supported in part by a research grant from the Faculty of Medicine of Tottori University (2008).

\section{REFERENCES}

1. Amann K, Rump LC, Simonaviciene A, Oberhauser V, Wessels S, Orth SR, Gross ML, Koch A, Bielenberg GW, Van Kats JP, Ehmke H, Mall G and Ritz E (2000) Effects of low dose sympathetic inhibition on glomerulosclerosis and albuminuria in subtotally nephrectomized rats. J Am Soc Nephrol 11, 1469-1478.

2. Borg J, Toazara J, Hietter H, Henry M, Schmitt G and Luu B (1987) Neurotrophic effect of naturally occurring longchain fatty alcohols on cultured CNS neurons. FEBS Lett 213, 406-410.

3. Borg J, Kesslak PJ and Cotman CW (1990) Peripheral administration of a long-chain fatty alcohol promotes septal cholinergic neurons survival after fimbria-fornix transection. Brain Res 518, 295-298.

4. Floege J, Alpers CE, Sage EH, Pritzl P, Gordon K, Johnson RJ and Couser WG (1992) Markers of complement-dependent and complement-independent glomerular visceral epithelial cell injury in vivo. Expression of antiadhesive proteins and cytoskeletal changes. Lab Invest 67, 486-497.

5. Gonzalez de Aguilar JL, Girlanda Junges C, Coowar D, Duportail G, Loeffler JP and Luu B (2001) Neurotrophic activity of 2,4,4-trimethyl-3-(15-hydroxypentadecyl)-2cyclohexen-1-one in cultured central nervous system neurons. Brain Res 920, 65-73.

6. Huang H, Shan J, Pan XH, Wang HP, Qian LB and Xia Q (2007) Carvedilol improved diabetic rat cardiac function depending on antioxidant ability. Diabetes Res Clin Pract 75, $7-13$.

7. Janssen U, Riley SG, Vassiliadou A, Floege J and Phillips AO (2003) Hypertension superimposed on type II diabetes in Goto Kakizaki rats induces progressive nephropathy. Kidney Int 63, 2162-2170.

8. Johnson RJ, Iida H, Alpers CE, Majesky MW, Schwartz SM, Pritzi P, Gordon K and Gown AM (1991) Expression of smooth muscle cell phenotype by rat mesangial cells in immune complex nephritis. Alpha-smooth muscle actin is a marker of mesangial cell proliferation. $J$ Clin Invest 87, 847858.

9. Kanetsuna Y, Takahashi K, Nagata M, Gannon MA, Breyer MD, Harris RC and Takahashi T (2007) Deficiency of endothelial nitric-oxide synthase confers susceptibility to diabetic nephropathy in nephropathy-resistant inbred mice. $\mathrm{Am} J$ Pathol 170, 1473-1484.

10. Kazuyama E, Saito M, Kinoshita Y, Satoh I, Dimitriadis F and Satoh K (2009) Endothelial dysfunction in the early- and late-stage type-2 diabetic Goto-Kakizaki rat aorta. Mol Cell Biochem 332, 95-102.

11. Kobayashi N (2002) Mechanism of the process formation; podocytes vs. neurons. Microsc Res Tech 57, 217-223.

12. Koya D, Haneda M, Nakagawa H, Isshiki K, Sato H, Maeda S, Sugimoto T, Yasuda H, Kashiwagi A, Ways DK, King GL and Kikkawa R (2000) Amelioration of accelerated diabetic mesangial expansion by treatment with a PKC beta inhibitor in diabetic $\mathrm{db} / \mathrm{db}$ mice, a rodent model for type 2 diabetes. FASEB $J$ 14, 439-447.

13. Mena-Martin FJ, Martin-Escudero JC, Simal-Blanco F, Carretero-Ares JL, Arzua-Mouronte D and Castrodeza Sanz JJ (2006) Influence of sympathetic activity on blood pressure and vascular damage evaluated by means of urinary albumin excretion. J Clin Hypertens (Greenwich) 8, 619-624.

14. Murakawa Y, Zhang W, Pierson CR, Brismar T, Ostenson CG, Efendic S and Sima AA (2002) Impaired glucose tolerance and insulinopenia in the GK-rat causes peripheral neuropathy. Diabetes Metab Res Rev 18, 473-483.

15. Nakagawa T, Sato W, Glushakova O, Heinig M, Clarke T, Campbell Thompson M, Yuzawa Y, Atkinson MA, Johnson RJ and Croker B (2007) Diabetic endothelial nitric oxide synthase knockout mice develop advanced diabetic nephropathy. J Am Soc Nephrol 18, 539-550.

16. Nobrega MA, Fleming S, Roman RJ, Shiozawa M, Schlick N, Lazar J and Jacob HJ (2004) Initial characterization of a rat model of diabetic nephropathy. Diabetes 53, 735-742.

17. Okada S, Saito M, Kazuyama E, Hanada T, Kawaba Y, Hayashi A, Satoh K and Kanzaki S (2008) Effects of $\mathrm{N}$-hexacosanol on nitric oxide synthase system in diabetic rat nephropathy. Mol Cell Biochem 315, 169-177.

18. Pagtalunan ME, Miller PL, Jumping-Eagle S, Nelson RG, Myers BD, Rennke HG, Coplon NS, Sun L and Meyer TW (1997) Podocyte loss and progressive glomerular injury in type II diabetes. J Clin Invest 99, 342-348.

19. Raij L, Azar S and Keane W (1984) Mesangial immune injury, hypertension, and progressive glomerular damage in Dahl rats. Kidney Int 26, 137-143.

20. Saeki T, Morioka T, Arakawa M, Shimizu F and Oite T (1991) Modulation of mesangial cell proliferation by endothelial cells in coculture. Am J Pathol 139, 949-957.

21. Saito M, Kinoshita Y, Satoh I, Shinbori C, Kono T, Hanada T, Uemasu J, Suzuki H, Yamada M and Satoh K (2006) $\mathrm{N}$-hexacosanol ameliorates streptozotocin-induced diabetic rat nephropathy. Eur J Pharmacol 544, 132-137.

22. Saito M, Kinoshita Y, Satoh I, Shinbori C, Suzuki H, Yamada M, Watanabe T and Satoh K (2007) Ability of cyclohexenonic long-chain Fatty alcohol to reverse diabetes-induced cystopathy in the rat. Eur Urol 51, 479-488.

23. Sato N, Komatsu K and Kurumatani H (2003) Late onset of diabetic nephropathy in spontaneously diabetic GK rats. $\mathrm{Am}$ $J$ Nephrol 23, 334-342.

24. Satoh I, Saito M, Kinoshita Y, Shomori K, Suzuki H, Yamada M, Kono T and Satoh K (2005) Effects of cyclohexenonic long-chain fatty alcohol on diabetic rat trachea. Life Sci 77, 2030-2039.

25. Shinbori C, Saito M, Kinoshita Y, Satoh I, Kono T, Hanada T, Nanba E, Adachi K, Suzuki H, Yamada M and Satoh K (2007) Cyclohexenonic long-chain fatty alcohol has therapeutic effects on diabetes-induced angiopathy in the rat aorta. Eur J Pharmacol 567, 139-144.

26. Watanabe $\mathrm{T}$ and Miyagawa I (2002) Effects of long-chain fatty alcohol on peripheral nerve conduction and bladder function in diabetic rats. Life Sci 70, 2215-2224.

27. Yagihashi S, Goto Y, Kakizaki M and Kaseda N (1978) Thickening of glomerular basement membrane in spontaneously diabetic rats. Diabetologia 15, 309-312.

28. Yajima G (1976) A histopathological study on diabetic nephropathy-light and electron microscopic observations. Acta Pathol Jpn 26, 47-62.

29. Zaoui P, Cantin JF, Alimardani-Bessette M, Monier F, Halimi 
S, Morel F and Cordonnier D (2000) Role of metalloproteases and inhibitors in the occurrence and progression of diabetic renal lesions. Diabetes Metab 26, 25-29.

30. Zhao HJ, Wang S, Cheng H, Zhang MZ, Takahashi T, Fogo AB, Breyer MD and Harris RC (2006) Endothelial nitric oxide synthase deficiency produces accelerated nephropathy in diabetic mice. J Am Soc Nephrol 17, 2664-2669.

31. Zhou X, Ono H, Ono Y and Frohlich ED (2002) N- and
L-type calcium channel antagonist improves glomerular dynamics, reverses severe nephrosclerosis, and inhibits apoptosis and proliferation in an L-NAME/SHR model. $J$ Hypertens 20, 993-1000.

32. Zou J, Yaoita E, Watanabe Y, Yoshida Y, Nameta M, Li H, Qu Z and Yamamoto T (2006) Upregulation of nestin, vimentin, and desmin in rat podocytes in response to injury. Virchows Arch 448, 485-492. 